\title{
Numerical Study of Correlation of Fluid Particle Acceleration and Turbulence Intensity in Swirling Flow
}

\author{
Nan Gui, ${ }^{1,2}$ Xingtuan Yang, ${ }^{1}$ Jie Yan, ${ }^{3}$ Jiyuan $\mathrm{Tu}^{1,4}$ and Shengyao Jiang ${ }^{1}$ \\ ${ }^{1}$ Institute of Nuclear and New Energy Technology of Tsinghua University and the Key Laboratory of \\ Advanced Reactor Engineering and Safety, Ministry of Education, Beijing 100084, China \\ ${ }^{2}$ Department of Thermal Energy Engineering, College of Mechanical and Transportation Engineering, \\ China University of Petroleum, Beijing 102249, China \\ ${ }^{3}$ China Academy of Space Technology, Beijing 10094, China \\ ${ }^{4}$ School of Aerospace, Mechanical \& Manufacturing Engineering, RMIT University, Melbourne, VIC 3083, Australia \\ Correspondence should be addressed to Shengyao Jiang; shengyaojiang@sina.com
}

Received 30 June 2014; Accepted 26 August 2014

Academic Editor: Junuthula N. Reddy

Copyright @ 2015 Nan Gui et al. This is an open access article distributed under the Creative Commons Attribution License, which permits unrestricted use, distribution, and reproduction in any medium, provided the original work is properly cited.

\begin{abstract}
Numerical investigation of correlation between the fluid particle acceleration and the intensity of turbulence in swirling flows at a large Reynolds number is carried out via direct numerical simulation. A weak power-law form correlation $u_{\mathrm{r} . \mathrm{m} . \mathrm{s}}^{E} \sim C\left(a^{L}\right)^{\varphi}$ between the Lagrangian acceleration and the Eulerian turbulence intensity is derived. It is found that the increase of the swirl level leads to the increase of the exponent $\varphi$ and the trajectory-conditioned correlation coefficient $\rho\left(a^{L}, u^{E}\right)$ and results in a weak power-law augmentation of the acceleration intermittency. The trajectory-conditioned convection of turbulence fluctuation in the Eulerian viewpoint is generally linearly proportional to the fluctuation of Lagrangian accelerations, indicating a weak but clear relation between the Lagrangian intermittency and Eulerian intermittency effects. Moreover, except the case with vortex breakdown, the weak linear dependency is maintained when the swirl levels change, only with the coefficient of slope varied.
\end{abstract}

\section{Introduction}

As well known, the swirling flow, especially for the strongly swirling jets at large Reynolds numbers to which few studies were devoted, is one type of anisotropic turbulence, which is less understood than the homogenous and isotropic turbulence $[1,2]$. In the past decades, the swirling flows have been studied extensively under the Eulerian framework, including a variety of scientific research topics and industrial applications, for example, swirling recirculating flow field [3, 4], open Von Kármán swirling flow [5], gas-liquid cylindrical cyclone [6], gas-liquid two-phase jet flow [7], gas turbine combustor [8], and so on. However, the swirling flows based on the Lagrangian framework have not been well investigated. Referring to the recent investigations of Lagrangian acceleration in highly turbulent flows $[9,10]$, the turbulent anisotropic flows with either a swirling or a rotating flow pattern are of specific interest. Unfortunately, the characteristics of Lagrangian acceleration for highly swirling flows are still not well known.

Moreover, due to the fundamental importance of the Lagrangian characteristics of fluid particle motion to transporting and mixing in turbulence and reacting systems [11], the Lagrangian measurement/modeling method has been well used recently as it is a powerful approach for improving the understanding of turbulent flows $[12,13]$. Therefore, a large number of excellent studies have been carried out contributing to the Lagrangian characteristics of fluid particle motion, especially on the acceleration structure or Lagrangian velocity spectrum [14-18].

On the other hand, as the investigations of turbulence under the Eulerian frame are better developed than those under the Lagrangian frame, establishing the relationship between the Lagrangian statistics and Eulerian statistics should be helpful in determining the common intrinsic statistical features of turbulence. However, the joint Lagrangian 
and Eulerian statistics of fluid particles are not well understood yet. For example, the relationship between acceleration intermittency (from the Lagrangian viewpoint) and turbulence intensity (from the Eulerian viewpoint) is unknown.

Additionally, to carry out direct numerical simulation of strongly swirling jets at a larger Reynolds number is a state-ofthe-art challenging work. Thus, direct numerical simulation of the strongly swirling jet is very essential and important to the scientific research of anisotropic turbulence.

In conclusion, a numerical study of direct numerical simulation of the Lagrangian motion of fluid particles at large Reynolds numbers is performed in present study, where the Lagrangian statistics and Eulerian statistics are combined together. The main aim of this study is to show the dependence of correlation between fluid particle acceleration and the intensity of turbulence on various levels of swirls and show the correlation between the Lagrangian acceleration and Eulerian convection of turbulence fluctuation in strongly swirling jets at a large Reynolds number.

\section{Numerical Description}

2.1. Governing Equations. The Navier-Stokes equations for incompressible fluids, based on the conservation laws of mass and momentum, are formulated in dimensionless forms as follows:

(1) continuum equation: $\partial u_{i} / \partial x_{i}=0$,

(2) momentum equation: $\left(\partial u_{i} / \partial t\right)+u_{j}\left(\partial u_{i} / \partial x_{j}\right)=$ $-\left(\partial p / \partial x_{i}\right)+(1 / \operatorname{Re})\left(\partial / \partial x_{j}\right)\left(\partial / \partial x_{j}\right) u_{i}$.

To solve the governing equations, the finite difference method is applied. The upwind compact schemes [19] are used to discretize the convection term. The fourth-order compact difference schemes [20] are applied for space derivatives and the pressure-gradient terms. The third-order explicit schemes are used to deal with the boundary points, keeping the global fourth-order spatial accuracy. The fourth-order Runge-Kutta schemes [21] are used for time integration. The pressurePoisson equation is solved to obtain the pressure via using the fourth-order finite difference method [22]. The methods have already been successfully applied in my recently published literature [23].

2.2. Simulation Setup. The parameters used in the present simulation are listed in Table 1 . In the momentum equation, the Reynolds number is defined as $\operatorname{Re}_{\lambda}=U_{0} \cdot \lambda / v=387.3$, where $U_{0}$ is the axial inlet velocity; $\lambda$ is the Taylor microscale; and the kinematic viscosity $v=10^{-6}\left(\mathrm{~m}^{2} / \mathrm{s}\right)$ (water at $\left.20.3^{\circ} \mathrm{C}\right)$.

The configurations of swirling flow are illustrated in Figure 1(a), which are similar to those from an earlier study [24]. A round jet of diameter $d$ is imparted with a rotational azimuthal velocity at the inlet and issued into a rectangular
TABLE 1: Parameters used in simulation.

\begin{tabular}{lc}
\hline Characteristic diameter $d^{*}(\mathrm{~mm})$ & 1.0 \\
Characteristic inflow velocity $U_{0}{ }^{*}(\mathrm{~m} / \mathrm{s})$ & 5,8 and 10 \\
Scales of the flow domain & $13.3 d \times 10 d \times 10 d$ \\
Grid numbers, $N_{x} \times N_{y} \times N_{z}(-)$ & $640 \times 480 \times 480$ \\
Spatial resolution $\delta(\mu \mathrm{m})$ & 20.8 \\
Reynolds number, $\mathrm{Re}$ & 10000 \\
Swirl number, $S$ & $0.49,1.08$ and 1.42 \\
Density of fluid, $\rho_{f}\left(\mathrm{~kg} / \mathrm{m}^{3}\right)$ & $1.0 \times 10^{3}$ \\
Kinematic viscosity of fluid, $\nu\left(\mathrm{m}^{2} / \mathrm{s}\right)$ & $1.0 \times 10^{-6}$ \\
Time step, $\Delta t(\mu \mathrm{s})$ & 0.2 \\
Total simulation time, $T_{s}(\mathrm{~ms})$ & 40 \\
\hline
\end{tabular}

${ }^{*}$ The characteristic variable.

flow domain of $13.3 d \times 10 d \times 10 d$. The swirl level is the ratio of the maximum azimuthal velocity $U_{a, \max }$ to the streamwise velocity $U_{0}$; that is, $S=2 U_{a, \max } / U_{0}$ [25]. The axial and azimuthal inlet velocity profiles are the same as those mentioned in earlier studies [24, 26, 27] (Figures 1(b) and $1(\mathrm{c})$ ). No inflow disturbance is introduced to keep the intrinsic feature of the strongly swirling flow. Three swirl numbers, that is, $S=0.49,1.08$, and 1.42 , are simulated in the present study by varying the azimuthal velocity. The nonreflecting boundary condition is utilized for the outlet condition [28], and the side walls are set to be nonslipping wall boundaries.

The flow domain is discretized by $640 \times 480 \times 480=$ 147456000 Cartesian mesh grids. With this spatial discretization $(\delta=20.8 \mu \mathrm{m})$, the jet inlet area $\left(\pi d^{2} / 4\right)$ is covered by 1791 mesh grids. It is estimated that the Kolmogorov length scale is about $\eta \approx 0.85 \delta$, which is in the same order of the finest mesh scale. According to [29], it is fine enough to capture the smallest scale of turbulence. For time integration, the time step is $0.2 \mu \mathrm{s}$ and the total simulated time is $40 \mathrm{~ms}$, about three convective periods.

The particles issued from the 1791 grid points inside the jet inlet area are traced, and, for each grid point, one particle is issued into the flow domain every 200 time steps. In this way, a total number of 179,100 fluid particles are traced in this study. The fluid particles originated from these 1791 points are designated as $\mathbf{x}(j, t), j=\bmod (n, 1791)$, where $n$ is the particle number and $j$ designates the group number. Then, all the particles can be divided into 1791 groups. Each group has the same initial location and velocity. However, the trajectories of fluid particles within each group are not the same because the system of fluid flow dynamics is strongly nonlinear. An ensemble average over each group of particles is utilized to carry out the Lagrangian statistics. For each group, the instantaneous acceleration for 100 particles is traced and recorded at any time for analysis. Thus, each data group includes $\sim O\left(10^{6}\right)$ points. The ensemble average process should be statistically reliable over such a large number of data points. 


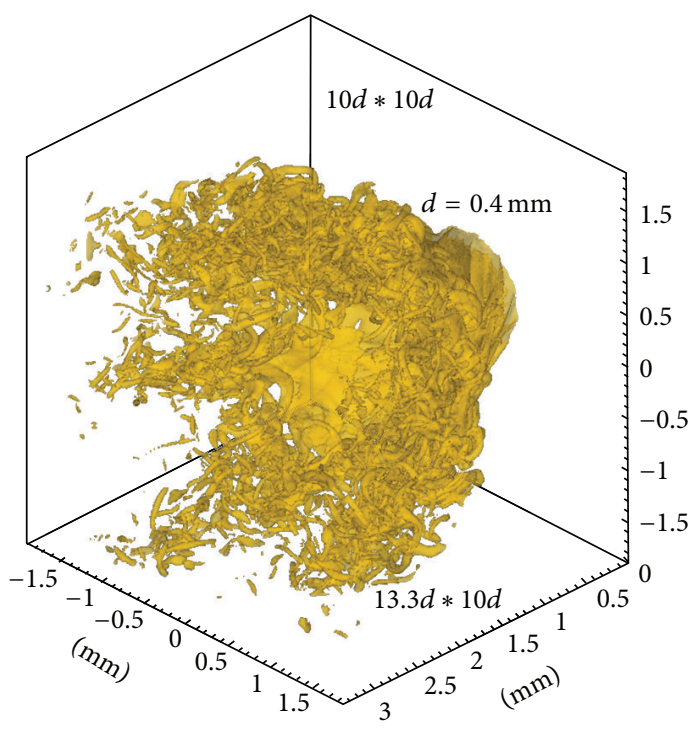

(a)

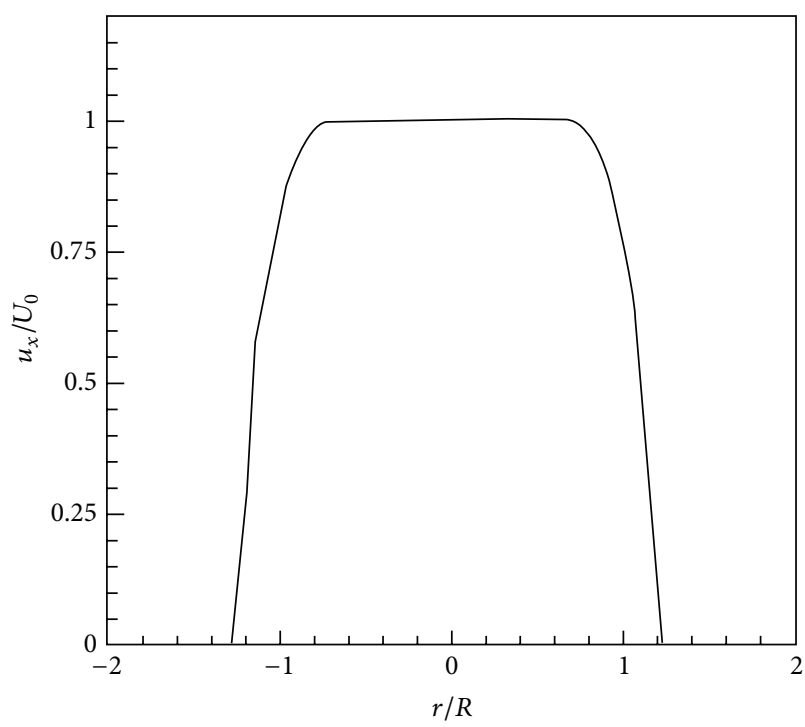

(b)

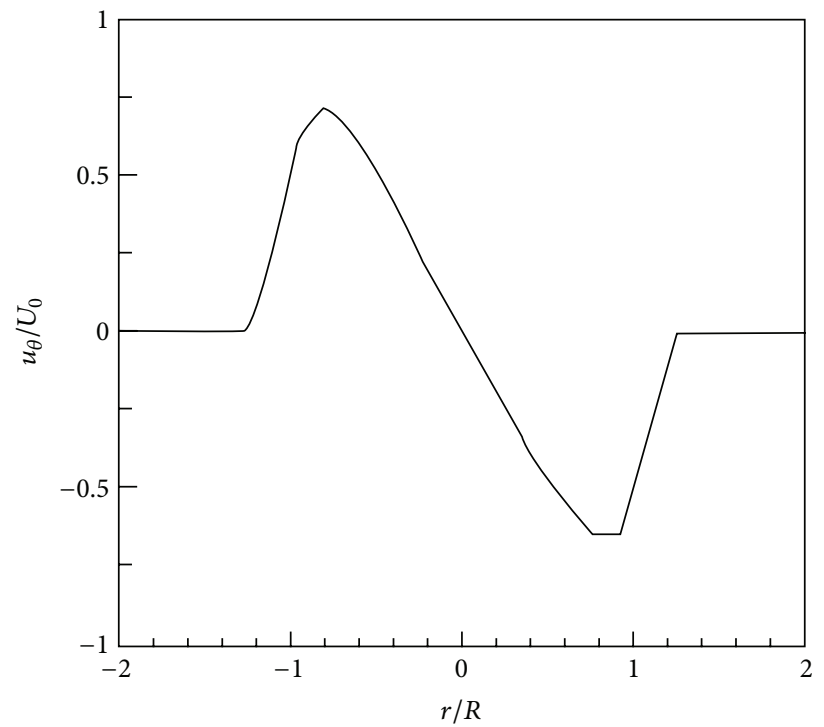

(c)

FIGURE 1: (a) Sketch of flow configuration and typical vorticity and the axial (b) and azimuthal (c) inlet velocity profiles.

\section{Results and Discussions}

3.1. Fluid Vortex Structures and Particle Trajectory. The typical vortex structure of $S=1.42$ is visualized in Figure 1(a). It is clearly seen that a bubble region is enclosed by the nearly axisymmetric vortices, forming an open angle toward downstream. It is so called the central recirculation zone (CRZ) or vortex breakdown (VB). Moreover, strong small vortices are observed due to the highly turbulent and swirling fluid flow.

Corresponding to this type of vortex structure, the fluid particles are transported from the initial locations to the downstream. The trajectories of one typical group of fluid particles originated from the same initial location at the inlet of the flow are shown in Figure 2. It is found that the fluid trajectories enclose a bubble region statistically too, which are initiated from the location where the axial motion of fluid particle is decreased to zero, that is, the stagnation point. After that, an immediate expansion in the lateral and spanwise directions is established. This feature of fluid trajectory reflects the structural characteristics of swirling flow under the Lagrangian viewpoint.

\subsection{Joint Distribution of Acceleration and Turbulence Intensity.} In general, the velocity field depends on the location and time, that is, $\mathbf{u}^{E}(\mathbf{x}, t)$, where " $E$ " denotes the "Eulerian" viewpoint. To average $\mathbf{u}^{E}(\mathbf{x}, t)$ in time, the velocity field can be divided into a time averaged part $\overline{\mathbf{U}}^{E}(\mathbf{x})$ and a fluctuation part $\mathbf{u}^{\prime E}(\mathbf{x}, t)$, corresponding to the mean flow field 


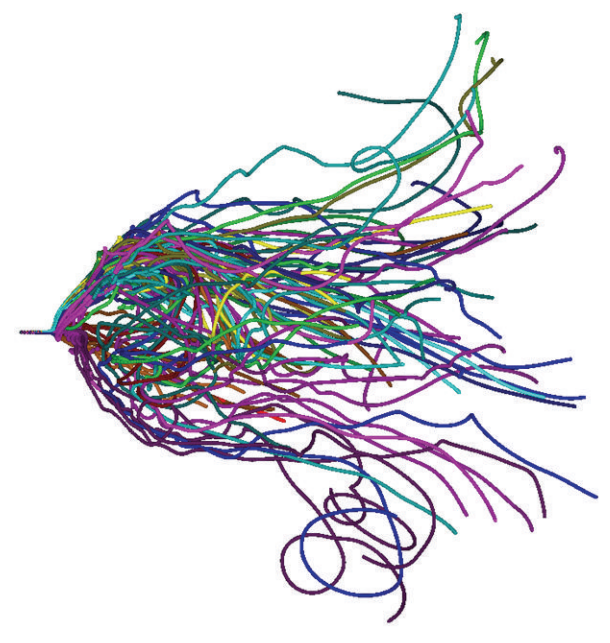

FIGURE 2: The trajectories of one group of particles for $S=1.42$.

and the turbulent fluctuation field, respectively. Hence, the root mean square (RMS) values of velocity $u_{\text {r.m.s }}^{E}(\mathbf{x})=$ $\left((1 / \Delta T) \int_{t}^{t+\Delta T}\left|\mathbf{u}^{E}(\mathbf{x}, \tau)-\overline{\mathbf{U}}^{E}(\mathbf{x})\right|^{2} d \tau\right)^{1 / 2}$ can be used to evaluate the local intensity of turbulence at location $\mathbf{x}$. On the other hand, the motion of fluid particles can be traced under the Lagrangian framework. The velocity and acceleration of the traced particles are defined as $\mathbf{a}^{L}(j, t)$ and $\mathbf{u}^{L}(j, t)$, respectively, where $j$ denotes the fluid particle and " $L$ " denotes the "Lagrangian" viewpoint. In this way, the statistical characteristics of Lagrangian motion of fluid particles with regard to the acceleration $\mathbf{a}^{L}(j, t)$, velocity $\mathbf{u}^{L}(j, t)$, and trajectories are obtained for analysis.

Assuming a "steady" turbulence field is reached, the turbulence intensity field can be quantified using the timeaveraging method. The fluid particle moves through the "steady" turbulence field with temporally and spatially varied Lagrangian acceleration of $a^{L}=a^{L}(j ; \mathbf{r}, t)$. Focusing on $u_{\text {r.m.s }}^{E}$ and $a^{L}=a^{L}(j ; \mathbf{r}, t)$ on the particle trajectories, a joint distribution of $\left(a^{L}(j ; t), u_{\text {r.m.s }}^{E}\right)$ can be obtained for analysis via the Eulerian-Lagrangian joint statistics of turbulence. It is termed as the trajectory conditioned joint distribution here.

As shown in Figure 3, the trajectory conditioned distribution of $\left(a^{L}(j ; t), u_{\mathrm{r}, \mathrm{s} s}^{E}\right)$ for different levels of swirl is indicated. Every point in Figure 3 illustrates the pair of $\left(a^{L}(j ; t), u_{\text {r.m.s }}^{E}\right)$ on the trajectories which the $j$ th group particles go through. Zonal distributions are observed for $S=0.49,1.08$, and 1.42, with the widths being increased under larger swirling levels. Thus, it is indicated that the $u_{\mathrm{r} . \mathrm{m} . \mathrm{s}}^{E}$ and $a^{L}(j)$ are correlated in some manner. Generally, this correlation may follow the form of power-law $y=a+x^{b}$. Note that the linear relationship is also included in the power-law, provided $b=1$. Based on this assumption, the data are fitted by the near power-law expression.

The $\log$ - $\log$ distribution of $a^{L}(j)$ and $u_{\text {r.m.s }}^{E}$ for $S=1.42$ is illustrated in Figure 4 . The trend of the $\log$ - $\log$ plot is approximately linear, although the data points are scattered widely. As the data points are corresponding to all fluid particles
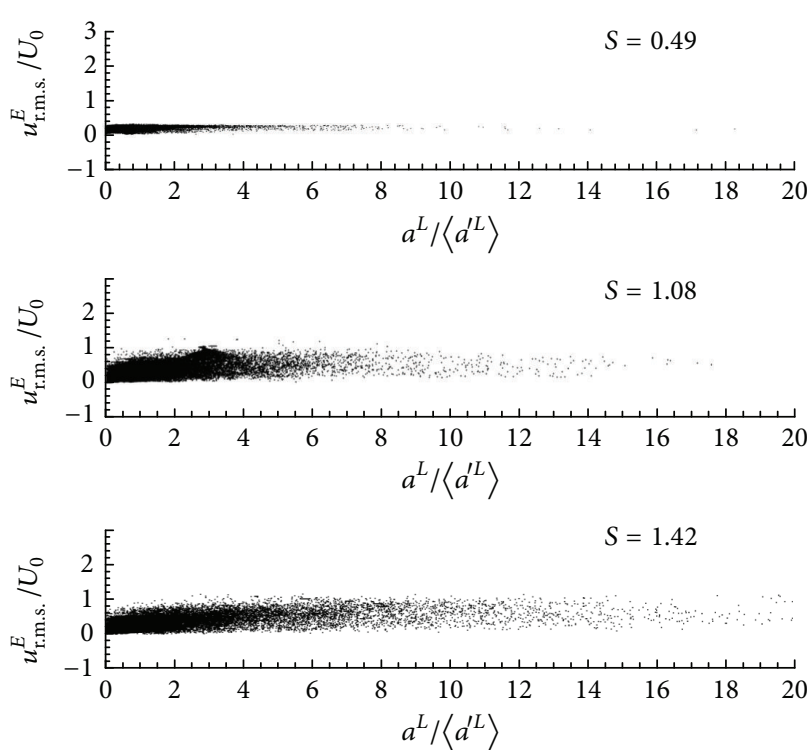

FIgURE 3: The distributions of Lagrangian acceleration $a^{L}$ and the Eulerian turbulence intensity $u_{\text {r.m.s }}^{E}$ for $S=0.49,1.08$, and 1.42 .

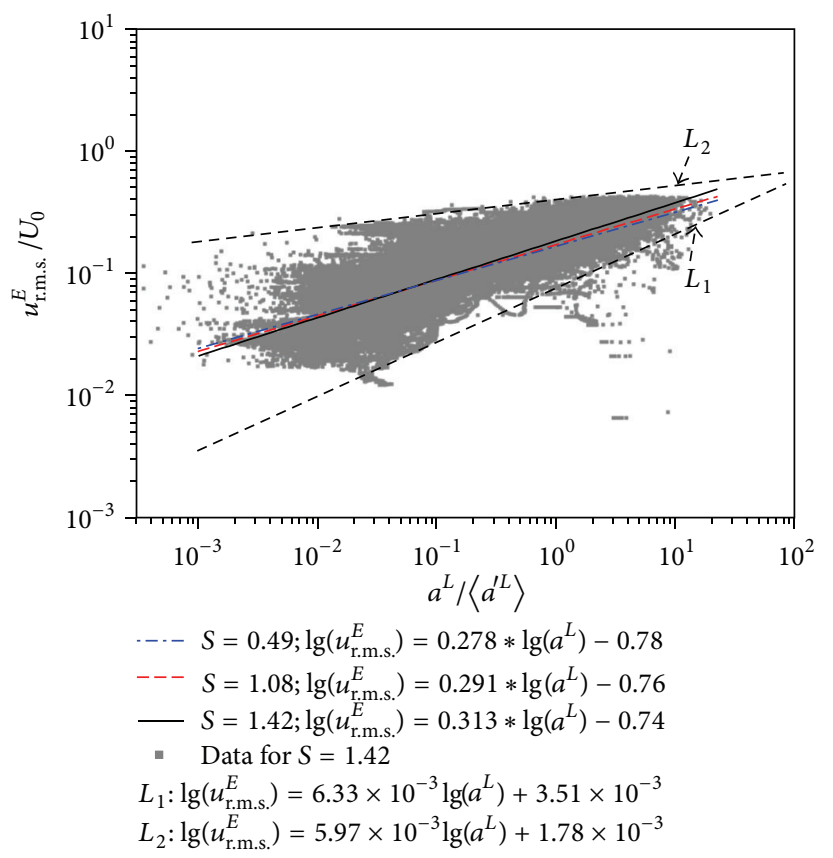

FIGURE 4: The fitting lines obtained by the linear regression of one group of fluid particles.

within the flow, the distribution should be scattered due to the stochastic and turbulent fluctuations of particles. Moreover, it could indicate the intrinsic Lagrangian characteristics of turbulent swirling flows, especially for the general trends of distribution and the statistical relationships between $a^{L}(j)$ and $u_{\text {r.m.s. }}^{E}$.

In addition, it is seen from Figure 4 that the slope of the fitting line increases when the swirl level increases. The fitting equations are listed in Table 2. By these fitted expressions, 


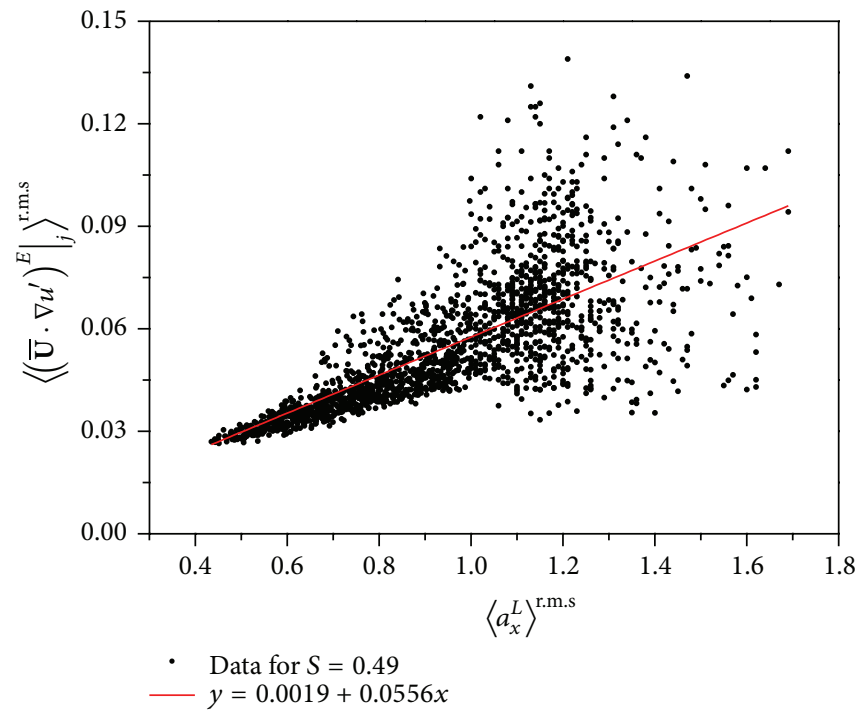

(a)

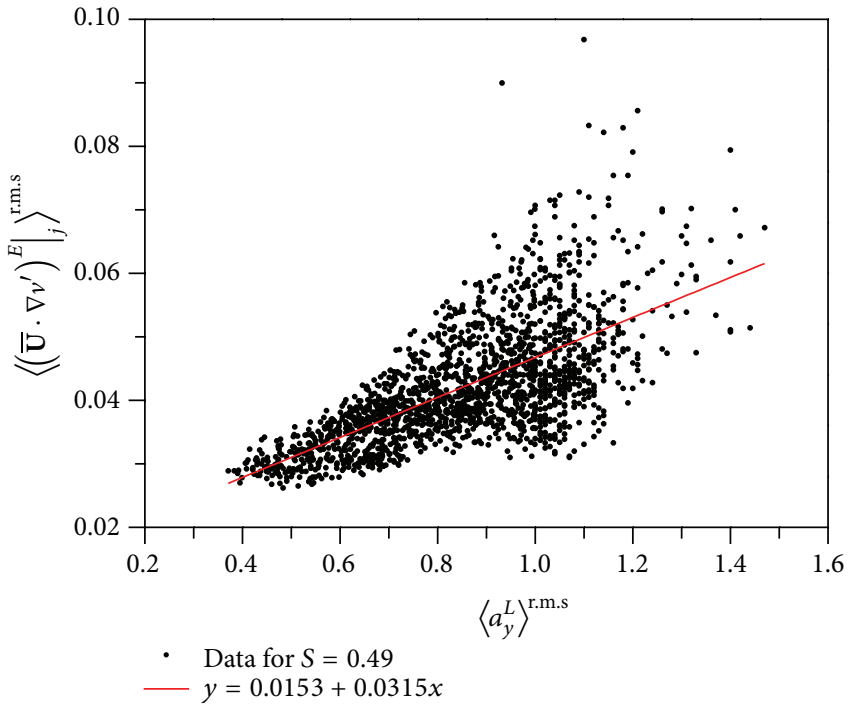

(b)

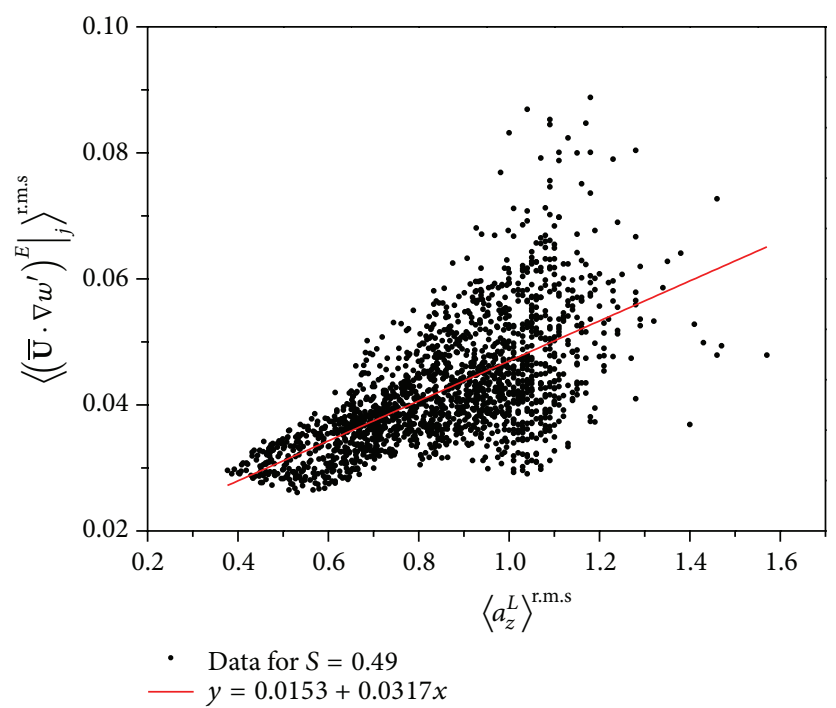

(c)

FiguRE 5: The correlation between the Eulerian convection term of turbulence fluctuations $u^{\prime}$ (a), $v^{\prime}$ (b), and $w^{\prime}$ (c) and the Lagrangian acceleration fluctuations in the $x, y$, and $z$ directions, respectively, for $S=0.49$.

the relationship between the Lagrangian acceleration and the local Eulerian turbulence intensity is indicated to follow a weak power-law form

$$
u_{\mathrm{r} . \mathrm{m} . \mathrm{s}}^{E} \sim C\left(a^{L}\right)^{\varphi} .
$$

Statistically, the turbulence intensity should be large where the fluid particles always pass through with large acceleration and vice versa. If the averaged turbulence intensity level is in the same order as that with the same Reynolds number and no extra disturbance, the effect of the swirl upon the Lagrangian characteristics could be achieved through a change in the exponent $\varphi$ and coefficient $C$. Hence, the characteristics of intermittency are closely related to swirl level and exponent $\varphi$. With the increase of swirl levels, the exponent $\varphi$ becomes larger. Consequently, the Lagrangian acceleration is nearly power-exponentially increased, leading to the augmented characteristics of intermittency.

In addition, Figure 4 shows that the scope of distribution of $\left(a^{L}(j ; t), u_{\text {r.m.s }}^{E}\right)$ can be generally sketched by an inferior line $\left(L_{1}\right)$ and a superior line $\left(L_{2}\right)$ (Table 3$)$. The majority of the data points are restricted inside the sketched scopes. The superior and inferior limiting lines show the proper limiting cases of the near power-law joint distribution; that is,

$$
1.0\left(\frac{a^{L}}{\left\langle a_{\mathrm{r} . \mathrm{m} . \mathrm{s}}^{\prime L}\right\rangle}\right)^{\alpha}<\left(\frac{u_{\mathrm{r} . \mathrm{m} . \mathrm{s}}^{E}}{U_{0}}\right)<1.5\left(\frac{a^{L}}{\left\langle a_{\mathrm{r} . \mathrm{m} . \mathrm{s}}^{\prime L}\right\rangle}\right)^{\alpha},
$$

where the limiting exponent is about $\alpha=6.16^{ \pm 0.17} \times 10^{-3}$. 


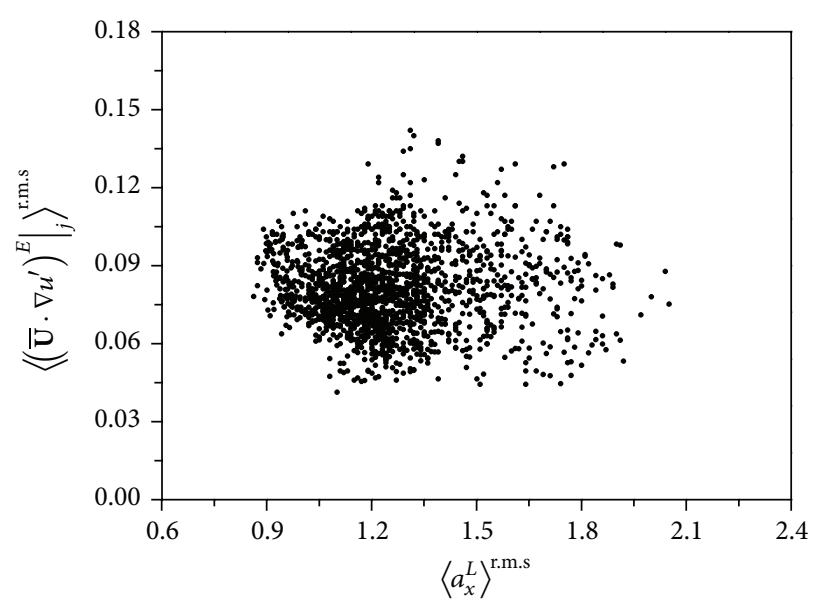

- Data for $S=1.42$

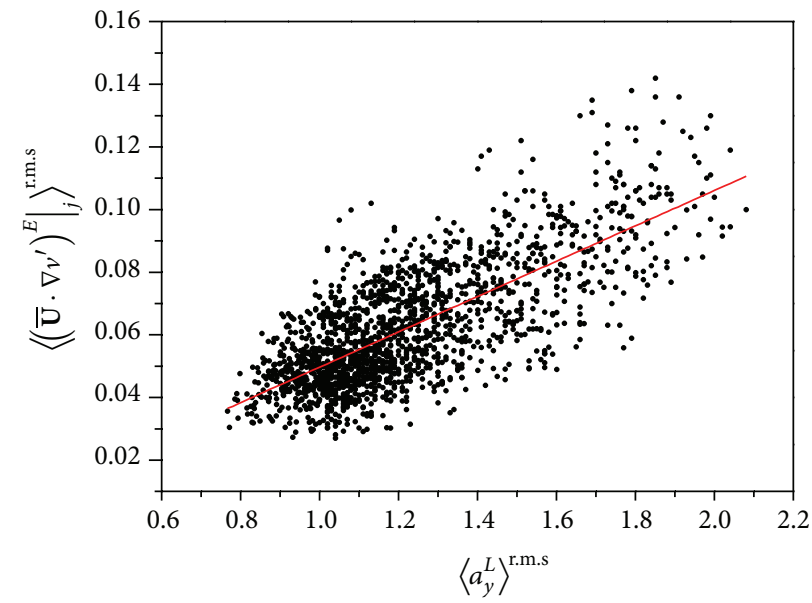

- Data for $S=1.42$

$-y=-0.0068+0.0564 x$

(b)

(a)

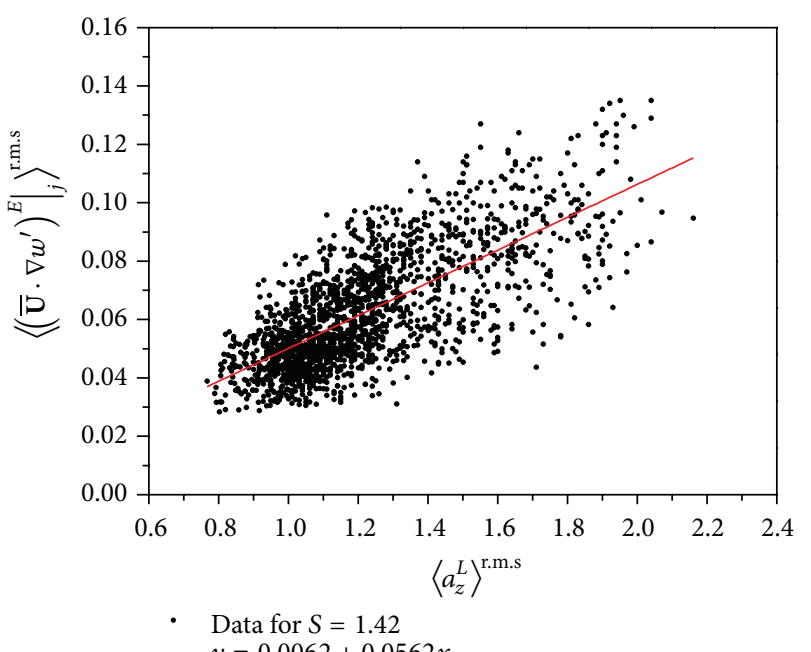

(c)

Figure 6: The correlation between the Eulerian convection term of turbulence fluctuations $u^{\prime}$ (a), $v^{\prime}$ (b), and $w^{\prime}$ (c) and the Lagrangian acceleration fluctuations in the $x, y$, and $z$ directions, respectively, for $S=1.42$.

TABLE 2: Fitting expressions.

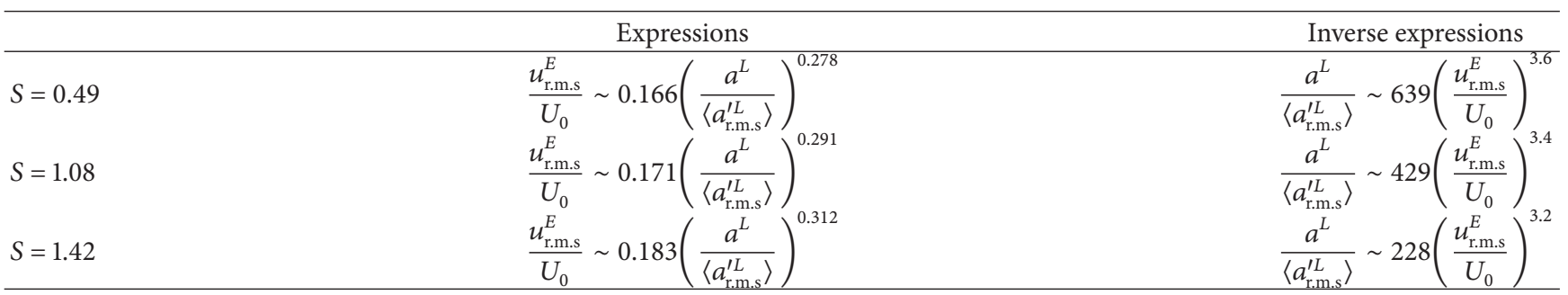

TABLE 3: Inferior and superior curves of the data points.

\begin{tabular}{lll}
\hline Inferior & $\lg \left(\frac{u_{\text {r.m.s }}^{E}}{U_{0}}\right)=6.332 \times 10^{-3} \lg \left(\frac{a^{L}}{\left\langle a_{\text {r.m.s }}^{\prime L}\right\rangle}\right)+3.514 \times 10^{-3}$ & $\left(\frac{u_{\text {r.m.s }}^{E}}{U_{0}}\right)=1.0\left(\frac{a^{L}}{\left\langle a_{\text {r.m.s }}^{\prime L}\right\rangle}\right)^{6.33 \times 10^{-3}}$ \\
Superior & $\lg \left(\frac{u_{\text {r.m.s }}^{E}}{U_{0}}\right)=5.974 \times 10^{-3} \lg \left(\frac{a^{L}}{\left\langle a_{\text {r.m.s }}^{\prime L}\right\rangle}\right)+1.777 \times 10^{-3}$ & $\left(\frac{u_{\text {r.m.s }}^{E}}{U_{0}}\right)=1.5\left(\frac{a^{L}}{\left\langle a_{\text {r.m.s }}^{\prime L}\right\rangle}\right)^{6.0 \times 10^{-3}}$ \\
\hline
\end{tabular}


3.3. Turbulent Convective Effect versus Lagrangian Acceleration. On the other hand, the Lagrangian acceleration is formulated as $D_{t}^{E}=\partial_{t}+\left(\mathbf{u}^{E} \cdot \nabla\right)$. With $\mathbf{u}^{E}=0$, the Lagrangian acceleration is equivalent to the Eulerian acceleration. Thus, the nonlinear convective term $\mathbf{u}^{E} \cdot \nabla$ plays a dominant role in correlation as well as the difference between the Eulerian statistics and the Lagrangian statistics of turbulence. Moreover, from the Eulerian viewpoint, $\mathbf{u}^{E}$. $\nabla=\overline{\mathbf{U}}^{E} \cdot \nabla+\mathbf{u}^{\prime E} \cdot \nabla$, where $\overline{\mathbf{U}}^{E}$ is the time-averaged velocity and $\mathbf{u}^{\prime E}$ is the fluctuation velocity. Based on the order analysis, it is assumed that the $\overline{\mathbf{U}}^{E} \cdot \nabla$ term plays a leading role in turbulence convection. Under this assumption, it is appropriate to consider that the term $\overline{\mathbf{U}}^{E} \cdot \nabla \mathbf{u}^{\prime E}$ represents the effect of convection of turbulence fluctuations. Remember that it is based on the Eulerian viewpoint. Then, moving with a Lagrangian fluid particle, it is natural to calculate the $\overline{\mathbf{U}}^{E} \cdot \nabla \mathbf{u}^{\prime E}$ along the particle trajectory. We call it the trajectory-conditioned $\overline{\mathbf{U}}^{E} \cdot \nabla \mathbf{u}^{\prime E}$, namely, $\left.\overline{\mathbf{U}}^{E} \cdot \nabla \mathbf{u}^{\prime E}\right|_{j}$. In this way, the joint Eulerian and Lagrangian statistics on correlation between the convection of turbulence fluctuation and the Lagrangian fluctuation of particle acceleration can be performed.

Figure 5 shows the RMS values of $\left.\overline{\mathbf{U}}^{E} \cdot \nabla \mathbf{u}^{\prime E}\right|_{j}$ and acceleration $\mathbf{a}^{L}$ for $S=0.49$ in the $x, y$, and $z$ directions, respectively. One point within Figure 5(a) represents the results for one group of particles. It is seen that, for low swirl levels $(S=$ 0.49 ), the RMS values are distributed nearly linearly. After linear regression, the fitted expression is $\left\langle\left.\overline{\mathbf{U}}^{E} \cdot \nabla u^{\prime E}\right|_{j}\right\rangle^{\text {r.m.s }} \approx$ $0.0556\left\langle a_{x}^{L}\right\rangle^{\text {r.m.s }}$. Moreover, it is observed from Figure 5(b) and Figure 5(c) that this nearly linear correlation also exists in the other directions; that is, $\left\langle\left.\overline{\mathbf{U}}^{E} \cdot \nabla v^{\prime E}\right|_{j}\right\rangle^{\text {r.m.s }} \approx 0.0315\left\langle a_{y}^{L}\right\rangle^{\text {r.m.s }}$ and $\left\langle\left.\overline{\mathbf{U}}^{E} \cdot \nabla w^{\prime E}\right|_{j}\right\rangle^{\text {r.m.s }} \approx 0.0317\left\langle a_{z}^{L}\right\rangle^{\text {r.m.s }}$. It indicates the correlation between the convection of turbulence fluctuations and the Lagrangian acceleration fluctuations along the particle trajectory; namely, the large fluctuation of Lagrangian acceleration indicates the large fluctuation of Eulerian convection of turbulence and vice versa. As a result, although scattered widely, the trends of the scattering distributions show the statistical correlation between the Lagrangian intermittency effects, which corresponds to the extreme events of the largely fluctuated Lagrangian acceleration, and the Eulerian intermittency effects of turbulence fluctuations, that is, the extreme events of convection of turbulence fluctuations.

However, for large swirl levels (Figure 6, $S=1.42$ ), this linear correlation in the axial direction seems to be disturbed, since the data points are widely scattered within a local domain. In contrast, the nearly linear correlation also occurs in the other directions. Remembering the occurrence of recirculation phenomena in the axial direction within the bubble-breakdown region, it is appropriate to explain that the disturbance of linear correlation is due to the formation of bubble vortex breakdown-a dramatic change of the large scale flow structure. In this way, the correlation of Lagrangian intermittency effects as well as the Eulerian intermittency effects becomes complex. It is correlated in the lateral and spanwise directions and attenuated in the axial direction.

\section{Conclusion}

The present study focuses on the effect of swirl levels on the correlation between the Lagrangian and Eulerian evaluations of turbulence. The statistical correlations between the Lagrangian and Eulerian evaluations of turbulence are demonstrated. It is found that the Lagrangian acceleration follows a weak power-exponential form of augmentation by the increase of swirl levels, leading to the augmented characteristics of intermittency. Moreover, the fluctuations of $a^{L}(\mathfrak{I}, t)$ and $u^{E}(\mathbf{r}, t)$ become increasingly correlated to each other with the increase of the swirl levels. Additionally, the Eulerian convection of turbulence fluctuations is weakly proportional to the Lagrangian acceleration fluctuations, indicating the proportionally close correlation between the Eulerian and Lagrangian intermittency effects.

\section{Conflict of Interests}

The authors declare that there is no conflict of interests regarding the publication of this paper.

\section{Acknowledgment}

The authors are grateful for the support of this research by the National Natural Science Foundation of China (Grant no. 51106180).

\section{References}

[1] J. Baldyga and J. R. Bourne, "Mixing and fast chemical reactionVIII: initial deformation of material elements in isotropic, homogeneous turbulence," Chemical Engineering Science, vol. 39, no. 2, pp. 329-334, 1984.

[2] J. S. Marshall and Y. Huang, "Simulation of light-limited algae growth in homogeneous turbulence," Chemical Engineering Science, vol. 65, no. 12, pp. 3865-3875, 2010.

[3] M. Freitag, M. Klein, M. Gregor et al., "Mixing analysis of a swirling recirculating flow using DNS and experimental data," International Journal of Heat and Fluid Flow, vol. 27, no. 4, pp. 636-643, 2006.

[4] A. Giannadakis, K. Perrakis, and T. Panidis, "A swirling jet under the influence of a coaxial flow," Experimental Thermal and Fluid Science, vol. 32, no. 8, pp. 1548-1563, 2008.

[5] T. Xing, "Direct numerical simulation of Open von Kármán Swirling Flow," Journal of Hydrodynamics, vol. 26, no. 2, pp. 165177, 2014.

[6] R. Hreiz, C. Gentric, and N. Midoux, "Numerical investigation of swirling flow in cylindrical cyclones," Chemical Engineering Research and Design, vol. 89, no. 12, pp. 2521-2539, 2011.

[7] G. A. Siamas, X. Jiang, and L. C. Wrobel, "Numerical investigation of a perturbed swirling annular two-phase jet," International Journal of Heat and Fluid Flow, vol. 30, no. 3, pp. 481-493, 2009.

[8] S. Tanaka, M. Shimura, N. Fukushima, M. Tanahashi, and T. Miyauchi, "DNS of turbulent swirling premixed flame in a 
micro gas turbine combustor," Proceedings of the Combustion Institute, vol. 33, no. 2, pp. 3293-3300, 2011.

[9] A. La Porta, G. A. Voth, A. M. Crawford, J. Alexander, and E. Bodenschatz, "Fluid particle accelerations in fully developed turbulence," Nature, vol. 409, no. 6823, pp. 1017-1019, 2001.

[10] H. G. Sun and W. Chen, "Fractal derivative multi-scale model of fluid particle transverse accelerations in fully developed turbulence," Science in China, Series E: Technological Sciences, vol. 52, no. 3, pp. 680-683, 2009.

[11] S. B. Pope, "Lagrangian PDF methods for turbulent flows," Annual Review of Fluid Mechanics, vol. 26, pp. 23-63, 1994.

[12] A. R. Rammohan, A. Kemoun, M. H. Al-Dahhan, and M. P. Dudukovic, "A lagrangian description of flows in stirred tanks via computer-automated radioactive particle tracking (CARPT)," Chemical Engineering Science, vol. 56, no. 8, pp. 2629-2639, 2001.

[13] G. Falkovich, K. Gawędzki, and M. Vergassola, "Particles and fields in fluid turbulence," Reviews of Modern Physics, vol. 73, no. 4, pp. 913-975, 2001.

[14] G. A. Voth, K. Satyanarayan, and E. Bodenschatz, "Lagrangian acceleration measurements at large Reynolds numbers," Physics of Fluids, vol. 10, no. 9, pp. 2268-2280, 1998.

[15] N. Mordant, A. M. Crawford, and E. Bodenschatz, "Threedimensional structure of the lagrangian acceleration in turbulent flows," Physical Review Letters, vol. 93, Article ID 214501, 2004.

[16] A. M. Crawford, N. Mordant, and E. Bodenschatz, "Joint statistics of the lagrangian acceleration and velocity in fully developed turbulence," Physical Review Letters, vol. 94, no. 2, Article ID 024501, 2005.

[17] N. Mordant, P. Metz, O. Michel, and J.-F. Pinton, "Measurement of lagrangian velocity in fully developed turbulence," Physical Review Letters, vol. 87, no. 21, Article ID 214501, 2001.

[18] L. Chevillard, S. G. Roux, E. Levêque, N. Mordant, J.-F. Pinton, and A. Arneodo, "Lagrangian velocity statistics in turbulent flows: effects of dissipation," Physical Review Letters, vol. 91, no. 21, Article ID 214502, 2003.

[19] D. X. Fu and Y. W. Ma, "A high order accurate difference scheme for complex flow fields," Journal of Computational Physics, vol. 134, no. 1, pp. 1-15, 1997.

[20] S. K. Lele, "Compact finite difference schemes with spectral-like resolution," Journal of Computational Physics, vol. 103, no. 1, pp. 16-42, 1992.

[21] A. Jameson and W. Schmidt, "Some recent developments in numerical methods for transonic flows," Computer Methods in Applied Mechanics and Engineering, vol. 51, no. 1-3, pp. 467-493, 1985.

[22] U. Anantha Krishnaiah, R. Manohar, and J. W. Stephenson, "Fourth-order finite difference methods for three-dimensional general linear elliptic problems with variable coefficients," Numerical Methods for Partial Differential Equations, vol. 3, no. 3, pp. 229-240, 1987.

[23] N. Gui, J. Yan, Z. Li, and J. Fan, "Direct numerical simulation of confined swirling jets," International Journal of Computational Fluid Dynamics, vol. 28, no. 1-2, pp. 76-88, 2014.

[24] N. Gui, J. Fan, and S. Chen, "Numerical study of particle-vortex interaction and turbulence modulation in swirling jets," Physical Review E: Statistical, Nonlinear, and Soft Matter Physics, vol. 82, no. 5, Article ID 056323, 2010.

[25] P. Billant, J.-M. Chomaz, and P. Huerre, "Experimental study of vortex breakdown in swirling jets," Journal of Fluid Mechanics, vol. 376, pp. 183-219, 1998.
[26] N. Gui, J. R. Fan, K. F. Cen, and S. A. Chen, "A direct numerical simulation study of coherent oscillation effects of swirling flows," Fuel, vol. 89, no. 12, pp. 3926-3933, 2010.

[27] N. Gui, J. R. Fan, and S. Chen, "Numerical study of particleparticle collision in swirling jets: a DEM-DNS coupling simulation," Chemical Engineering Science, vol. 65, no. 10, pp. 32683278, 2010.

[28] I. Orlanski, "A simple boundary condition for unbounded hyperbolic flows," Journal of Computational Physics, vol. 21, no. 3, pp. 251-269, 1976.

[29] P. Moin and K. Mahesh, "Direct numercial simulation: a tool in turbulence research," Annual Review of Fluid Mechanics, vol. 30, pp. 539-578, 1998 . 


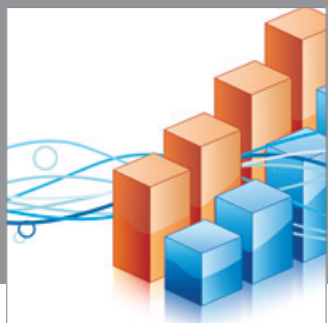

Advances in

Operations Research

mansans

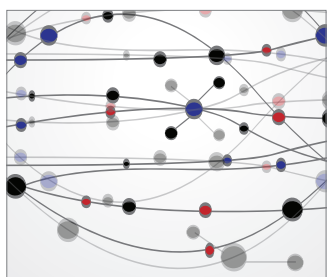

The Scientific World Journal
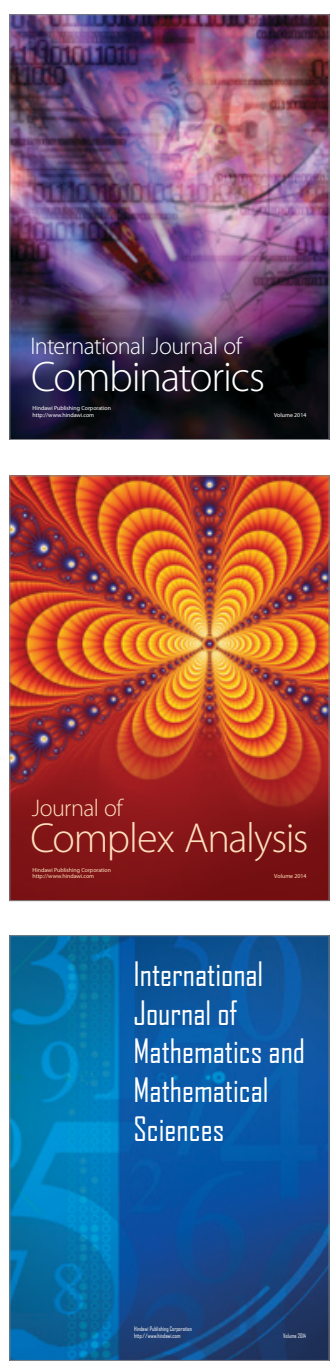
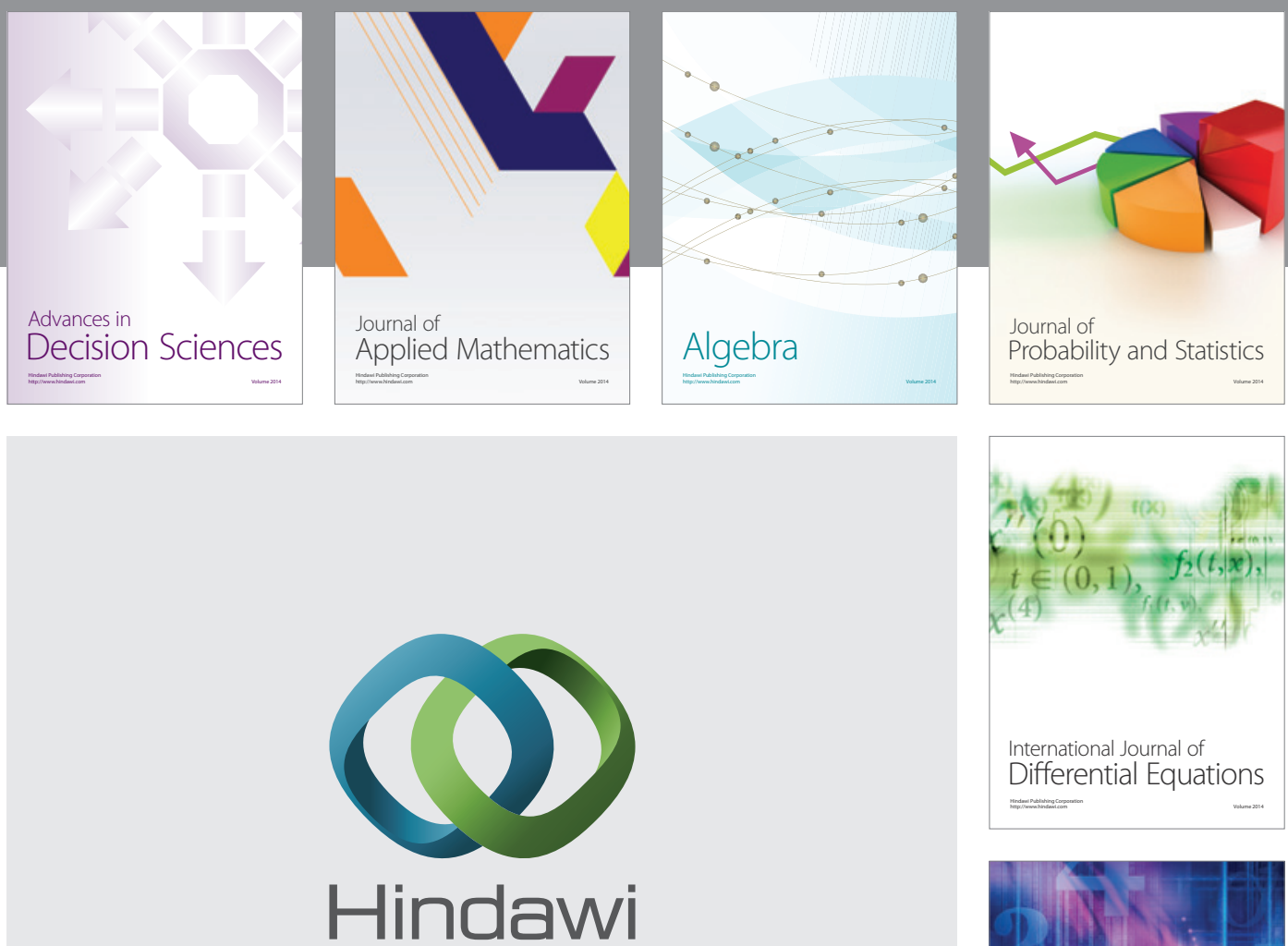

Submit your manuscripts at http://www.hindawi.com
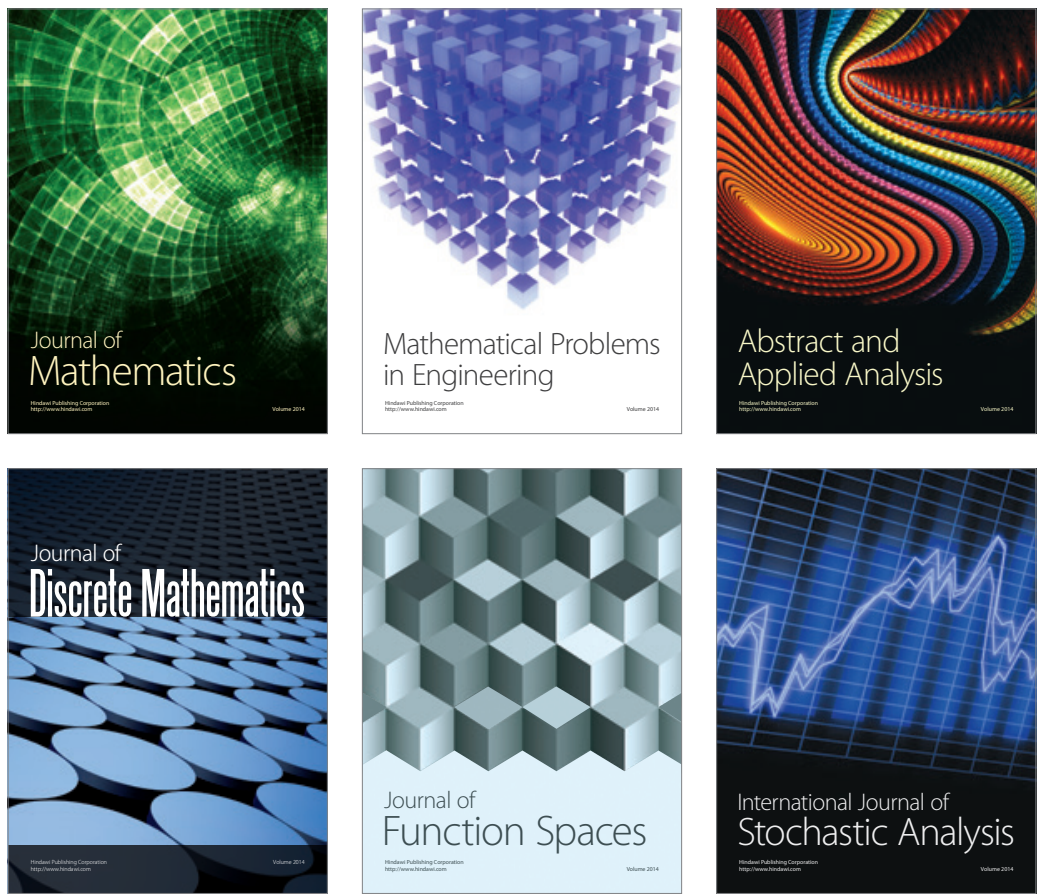

Journal of

Function Spaces

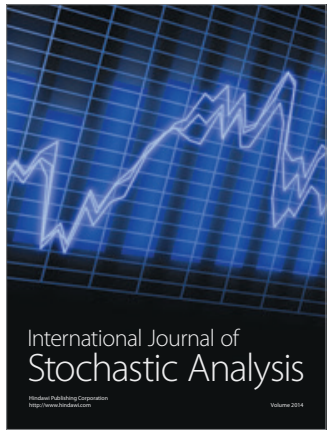

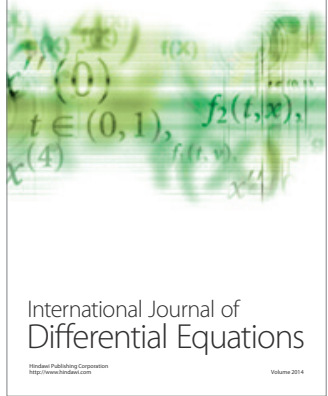
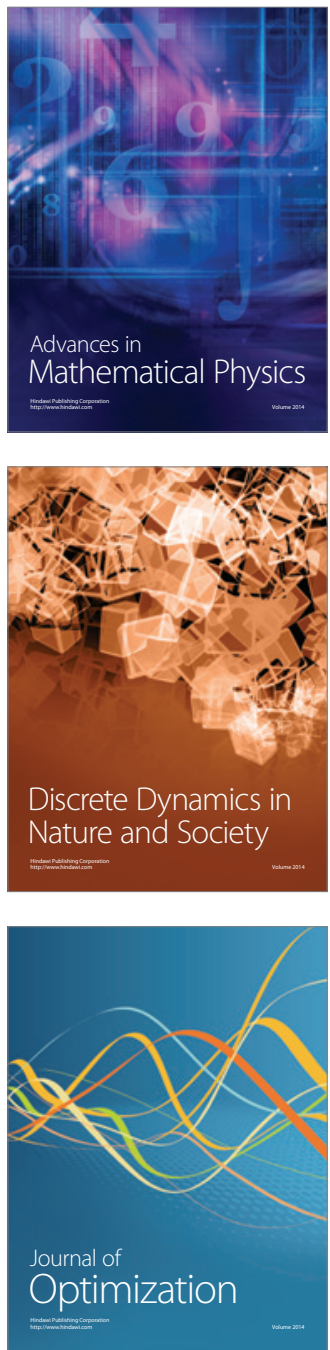